\title{
Deep multi-region whole-genome sequencing reveals heterogeneity and gene-by-environment interactions in treatment-naive, metastatic lung cancer
}

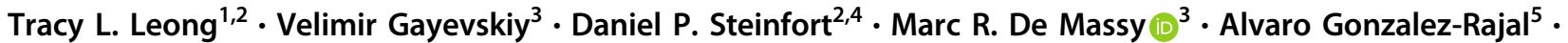 \\ Kieren D. Marini ${ }^{6} \cdot$ Emily Stone $^{6,7}$ - Venessa Chin ${ }^{5,8,9} \cdot$ Adrian Havryk $^{7,8}$ - Marshall Plit ${ }^{7,8} \cdot$ Louis B. Irving $^{2,4}$. \\ Barton R. Jennings ${ }^{10} \cdot$ Rachael A. McCloy ${ }^{5} \cdot$ W. Samantha N. Jayasekara ${ }^{6}$ Muhammad Alamgeer $^{6} \cdot$ Vishal Boolell $^{6}$. \\ Andrew Field ${ }^{8,11}$. Prudence A. Russell ${ }^{12}$ - Beena Kumar ${ }^{13}$ - Daniel J. Gough ${ }^{6,14}$ • Anette Szczepny ${ }^{6}$. \\ Vinod Ganju $\mathbb{1}^{6,14}$ • Fernando J. Rossello $\mathbb{1}^{15,16}$. Jason E. Cain ${ }^{6,14}$ • Anthony T. Papenfuss ${ }^{17,18,19}$. \\ Marie-Liesse Asselin-Labat id ${ }^{1,2}$ - Mark J. Cowley ${ }^{3,8,20} \cdot$ D. Neil Watkins M $^{5,7,8,9}$
}

Received: 8 July 2018 / Revised: 13 September 2018 / Accepted: 19 September 2018 / Published online: 22 October 2018

(c) The Author(s) 2018. This article is published with open access

\begin{abstract}
Our understanding of genomic heterogeneity in lung cancer is largely based on the analysis of early-stage surgical specimens. Here we used endoscopic sampling of paired primary and intrathoracic metastatic tumors from 11 lung cancer patients to map genomic heterogeneity inoperable lung cancer with deep whole-genome sequencing. Intra-patient heterogeneity in driver or targetable mutations was predominantly in the form of copy number gain. Private mutation signatures, including patterns consistent with defects in homologous recombination, were highly variable both within and between patients. Irrespective of histotype, we observed a smaller than expected number of private mutations, suggesting that ancestral clones accumulated large mutation burdens immediately prior to metastasis. Single-region whole-genome sequencing of from 20 patients showed that tumors in ever-smokers with the strongest tobacco signatures were associated with germline variants in genes implicated in the repair of cigarette-induced DNA damage. Our results suggest that lung cancer precursors in ever-smokers accumulate large numbers of mutations prior to the formation of frank malignancy followed by rapid metastatic spread. In advanced lung cancer, germline variants in DNA repair genes may interact with the airway environment to influence the pattern of founder mutations, whereas similar interactions with the tumor microenvironment may play a role in the acquisition of mutations following metastasis.
\end{abstract}

\section{Introduction}

Genomic heterogeneity is now recognized as a major challenge to the success of conventional therapy, targeted

These authors contributed equally: Tracy L. Leong, Velimir Gayevskiy

Electronic supplementary material The online version of this article (https://doi.org/10.1038/s41388-018-0536-1) contains supplementary material, which is available to authorized users.

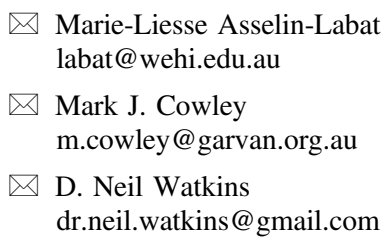

Extended author information available on the last page of the article therapy, and immunotherapy in the treatment of cancer [1]. The advent of multi-region sequencing has led to the identification of a previously unknown degree of complexity and genomic heterogeneity in solid tumors [2]. These landmark findings have major implications for the understanding of tumor initiation and evolution, particularly with respect to the development of metastasis [3]. Furthermore, they illustrate how analyzing multiple tumor sites from a relatively small number of cases can reveal the complexities of genomic evolution that cannot be resolved by single-site sequencing of large cohorts [1].

Although conclusions vary in different tumor systems, the use of genomic data to model tumor phylogenies suggests that metastasis can occur early in the life of the primary tumor [4]. There is also debate as to whether the emergence of private mutations in metastatic disease drives metastasis from rare clones present in the primary tumor, are the result of local environmental pressures unique to 
each metastatic site, or are random passenger events [5]. The propensity of lung cancer to spread to local and regional lymph nodes or beyond is reflected in the small numbers of patients eligible for curative surgery, and the lack of success in treating inoperable, locally advanced tumors with radical chemo-radiotherapy. Lung cancer represents a major challenge with respect to mapping genomic heterogeneity, in that most patients are inoperable at diagnosis [6]. Since surgical specimens offer high-quality tissue samples, early-stage lung cancers have largely formed the basis of large-scale sequencing projects in lung cancer [7-9], a better understanding of genomic heterogeneity in advanced lung cancer may inform the successful deployment of targeted therapy and immunotherapy.

Reflecting these technical barriers, multi-region sequencing analysis of lung cancer has been largely limited to surgically resected specimens $[10,11]$. In this regard, access to high-quality specimens from metastatic lymph nodes in surgical cases is limited, since the entire node is required for accurate diagnosis and staging rather than research. Therefore, the degree to which genomic heterogeneity might contribute to the pathogenesis of treatment-naive, metastatic lung cancer remains an open question. To address this uncertainty, we combined two approaches that circumvented the need to rely on surgical specimens. First, we obtained samples from newly diagnosed lung cancer patients undergoing endobronchial ultrasound-guided transbronchial needle aspiration (EBUS-TBNA), a minimally invasive technique that yields high-quality samples from both the primary tumor and intrathoracic lymph nodes in the same patient [12]. Second, we analyzed these specimens using high-depth whole-genome sequencing (WGS), which provides advantages over whole-exome sequencing (WES) in cancer specimens with respect to uniformity of coverage, high-resolution detection of copy number variation $(\mathrm{CNV})$ and structural variation (SV) $[13,14]$, as well as genome-wide, unbiased assessment of somatic mutation signatures [15]. Here we apply the principles of multiregion sequencing to metastatic lung adenocarcinoma (LUAD), squamous cell carcinoma (LUSC), and small cell lung cancer (SCLC), the three commonest forms of the disease.

\section{Results}

\section{Intra-patient heterogeneity in metastatic lung cancer}

A total of 30 primary and metastatic samples from 11 patients undergoing EBUS-TBNA for the diagnosis and/or staging of lung cancer were deemed informative based on tumor cellularity estimates of cytology smears and DNA quality (Fig. 1). Except for one sample, all were analyzed with WGS to an average depth of $150 \times$, including two cases in which two samples were obtained from the primary tumor in addition to the metastases. Peripheral blood mononuclear cells were used as a germline control sequenced to an average depth of 30-40 $\times$. In addition, a further nine patients yielded informative samples from a single site and were sequenced to an average WGS depth of $60 \times($ Supplementary Figure S1).

When comparing primary with metastatic tumors, mutations in known lung cancer driver genes (Supplementary Data File S1) such as TP53, KRAS, FAT1, PTEN, and $R B 1$, as well as homozygous deletions in $C D K N 2 A$ and $R B 1$, were conserved across all samples in individual patients (Fig. 1; Supplementary Data File S3). Exceptions included events private to the primary tumor (SETD2 and ARIDIA in LUAD6; NOTCH2 in SCLC1) (Fig. 1), or private to metastases (SMARCA4 in LUAD5; ARID1A in LUAD7) (Fig. 1). Heterogeneity was more prevalent with respect to copy number gain, with private amplification events in driver genes including MYC, CCND3, FGFR1, and TP63 (Fig. 1; Supplementary Data File S3). Several of these amplification events were private to the primary tumor, rather than the associated metastasis (Fig. 1). This pattern of heterogeneity was similar when considering a broader list of pan-cancer driver genes (Supplementary Data File S1), with private amplification events seen in ERBB3, RHEB, SOS1, SOS2, and EZH2 (Supplementary Figure S2).

We observed mutations in several important pan-cancer drivers not normally associated with lung cancer, including TSC1 in LUAD1 and LUSC5, and WT1 in LUAD6 and LUAD12. (Supplementary Figure S3). In addition, we detected medium and high-impact missense mutations associated with somatic loss-of-heterozygosity ( $\mathrm{LOH}$ ) in several novel genes with potential functional significance (Supplementary Table S1; Supplementary Figure S3). These included loss-of-function (LOF) variants in the Wilson's disease copper exporter $A T P 7 B$ (LUAD1), the Tolllike receptor TLR4 (LUAD13), and in ERAP2 (LUAD1), whose gene product promotes the presentation of endogenous cellular peptides by major histocompatibility complex class I molecules (Supplementary Table S1). Two potential gain-of-function (GOF) variants were also identified, one in the kinase domain of JAK2 in LUAD4, the other in the growth factor receptor domain of ERBB4 in LUSC4 (Supplementary Figure S3b).

Overall, these data are in keeping with similar results in metastatic pancreatic cancer [16], and suggest that there is limited heterogeneity in metastatic lung cancer with respect to mutations in key driver genes, and are consistent with a model in which these critical events are required for both the initiation and progression of the primary tumor prior to metastasis. 


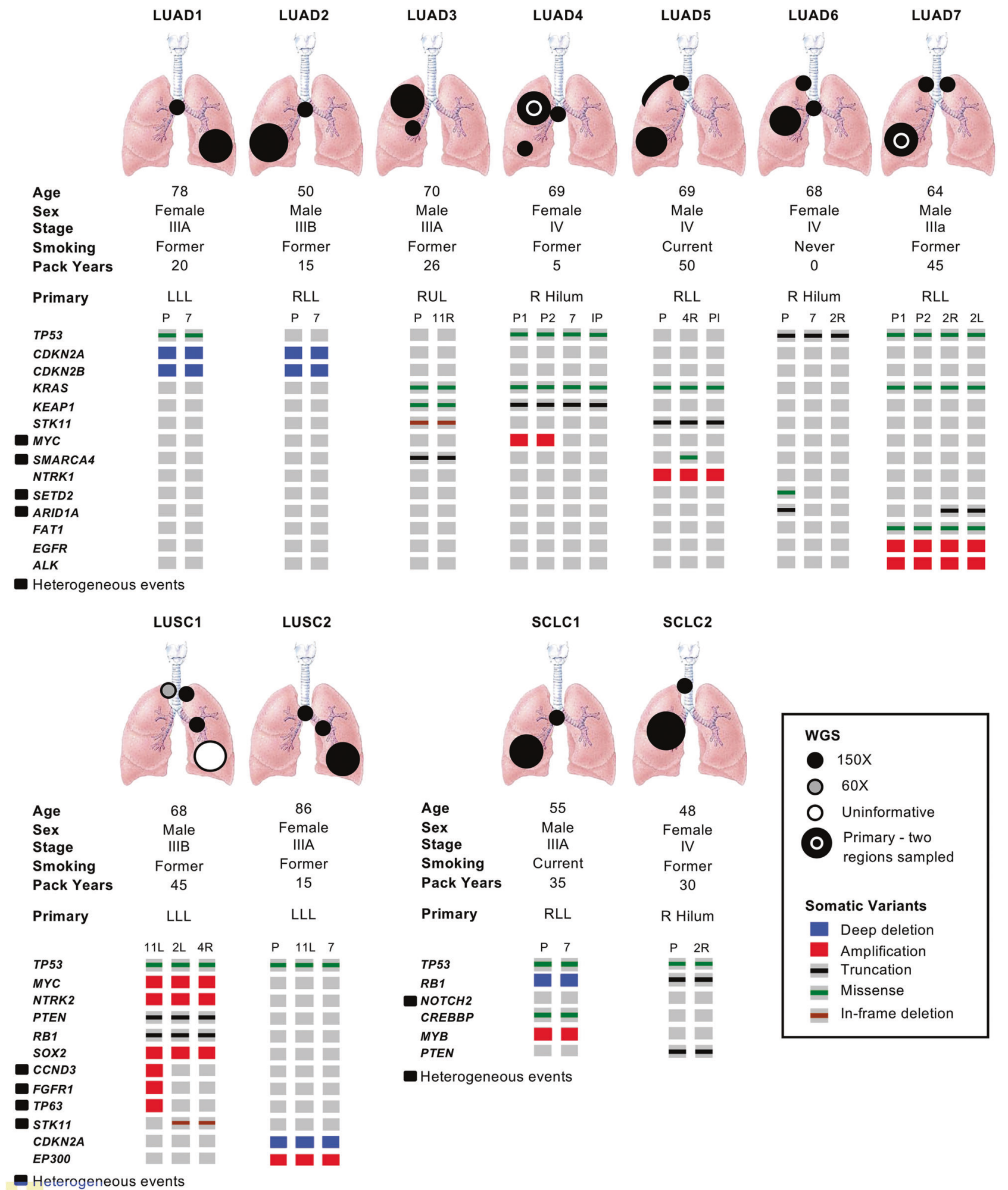

Fig. 1 Overview of cases analyzed with multi-region whole-genome sequencing. Oncoprint data depicting single-nucleotide variants, insertion/deletions, and copy number events in known lung cancer driver genes for each sample are shown with heterogeneous mutations labeled. LUAD lung adenocarcinoma, LUSC lung squamous cell carcinoma, SCLC small cell lung cancer, P primary tumor, IP intrapulmonary, Pl pleural. Metastases are otherwise indicated by lymph node station 

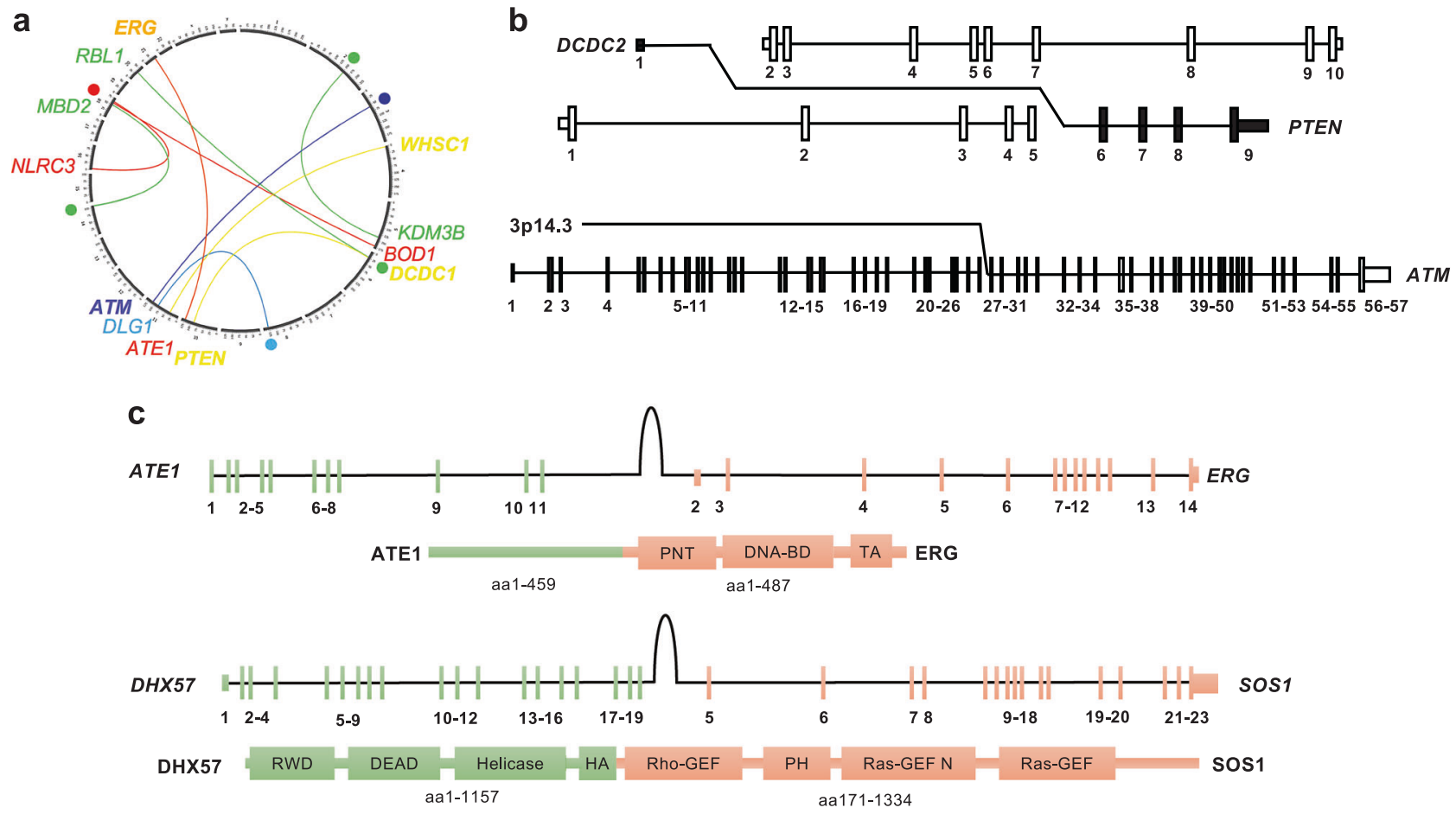

Fig. 2 Novel fusion events. a Circos plot depicting examples of novel fusion events. Each case is represented by one color. Intergenic regions are indicted by colored circles. b Examples of candidate lossof-function fusion events in the tumor-suppressor genes PTEN and

ATM. c Models of candidate gain-of-function fusion events depicted at genome and protein level. Protein domains are labeled according to Pfam convention. Exons in each fusion partner are numbered

\section{Novel fusions}

Fusions are now recognized as important GOF driver events in solid tumors, including well-described targetable events in genes such as $A L K$, ROSl, and NTRK1 in LUAD [17]. However, LOF fusion events in solid tumors are less well characterized. In the 20 cases analyzed, we detected three fusion events in known cancer driver genes (ATM in LUSC2; PTEN in LUSC1; WHSC1 in LUSC1), and a further seven events in candidate tumor-suppressor genes, including BOD1, DLG2, MBD2, and RBL1 (Fig. 2a,b; Supplementary Figure S4; Supplementary Table S2). In each case, the predicted transcript resulted in truncated or nonsense gene products (Supplementary Table S2). In addition, we identified two candidate GOF fusion events (Fig. 2a,c; Supplementary Table S2; Supplementary Data File S3). In SCLC1, we detected a candidate fusion of the N terminus of the arginyltransferase ATEl to the pointed (PNT), DNA binding and transactivation domains of ERG, an ETS-family transcription factor subject to frequent GOF fusion events in prostate cancer [18]. In LUAD7, we detected a candidate fusion event involving the $\mathrm{N}$ terminus of the DEAH-Box Helicase DHX57 with the catalytic C terminus domain of the RAS guanine nucleotide exchange factor SOS1 (Fig. 2a,c). The detection of 12 novel fusions in only 20 cases of advanced lung cancer reflects the sensitivity of WGS in detecting such events, and suggests that pathogenic fusions may be much more common in advanced lung cancer than previously appreciated. The potential for fusions to inactivate critical tumor-suppressor genes is supported by the recent description of LOF rearrangements in TP53 in osteosarcoma [19], and suggests that such events may add to mutation, deletion, and epigenetic gene silencing as an important path of tumor-suppressor inactivation.

\section{Somatic mutation signatures and germline variants in DNA repair genes}

We compared the mutation burden and somatic signatures shared by all tumor samples in each multi-region case, and in the informative samples of single-site cases, with smoking history (Fig. 3a). As expected, tumors in the 4 current smokers, each with over 20 pack-years exposure, were hypermutated ( $>10$ variants/Mb), and were dominated by a strong tobacco-related somatic signature (Fig. 3a). In keeping with published work, we also observed low mutation burdens in the tumors of the 3 never-smokers, which were dominated by Apolipoprotein B MRNA Editing Enzyme Catalytic Subunit 3G (APOBEC) and 5-methylcytosine (5-mC) deamination patterns typically associated with age or inflammation [20] (Fig. 3a). Tumors in the 13 
a

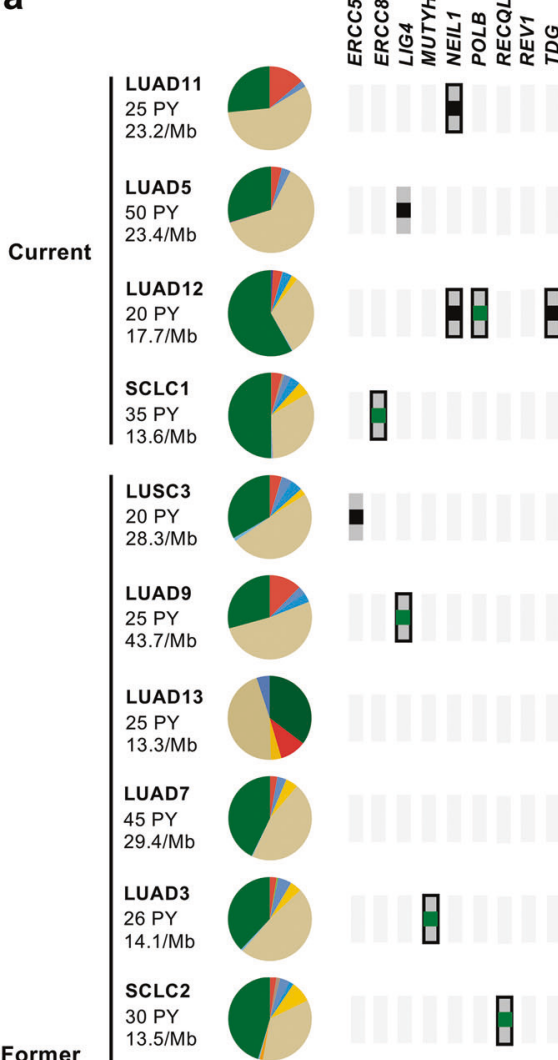

$13.5 / \mathrm{Mb}$

LUSC1

$45 \mathrm{PY}$

$20.8 / \mathrm{Mb}$

LUAD4

$5 \mathrm{PY}$

Lusc2

$15 \mathrm{PY}$

$17.71 / \mathrm{Mb}$

LUSC5

$18 \mathrm{PY}$

$9.26 / \mathrm{Mb}$

LUSC4

$20 \mathrm{PY}$
$17.94 / \mathrm{Mb}$

LUAD1

$20 \mathrm{PY}$

$7.24 / \mathrm{Mb}$

LUAD2

$15 \mathrm{PY}$

3.32/Mb

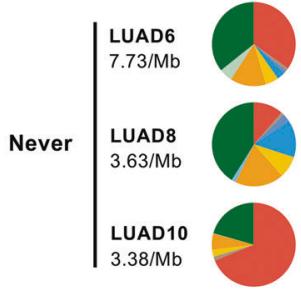

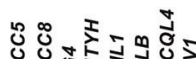

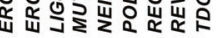

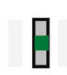

POLB

336 aа

MUTYH

536 aa

Alkylating Agents

APOBEC

Deamination $5 \mathrm{mC}$

HR Defect

MMR Defect

POLE

Smoking

- Tobacco Chewing

Transcription Strand Bias T>A

- Unknown

UV Light

$\prod$ Germline variant

Somatic LOH

- Truncation

- Missense

b

C
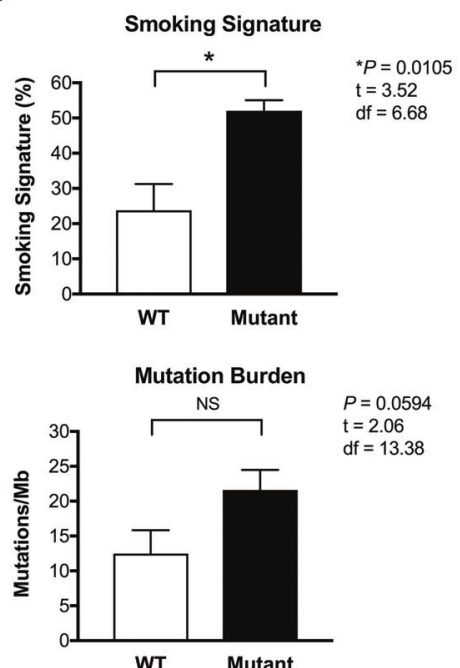

ERCC8

397 aa
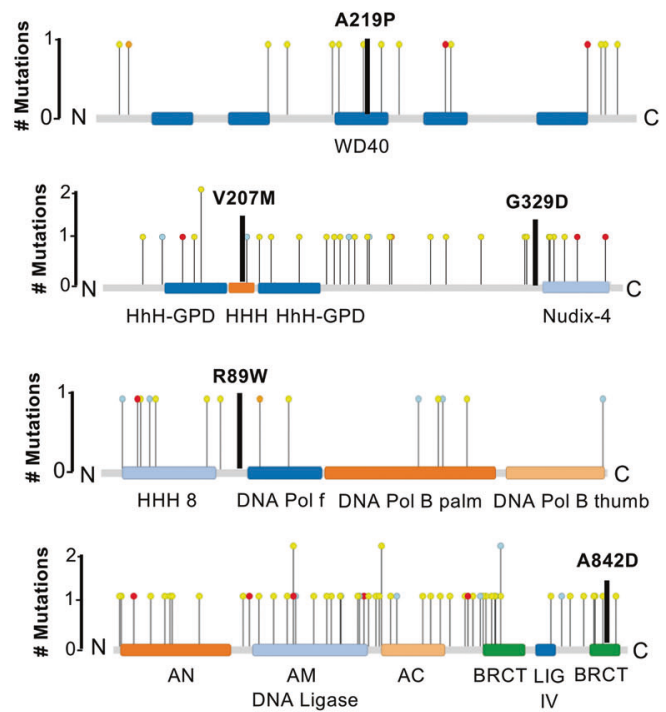

NEIL1

391 aa

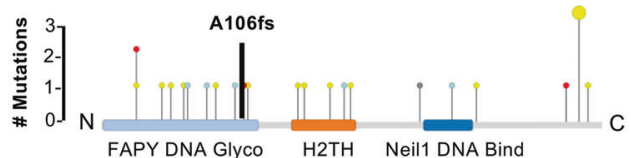

REV1

1252 aa

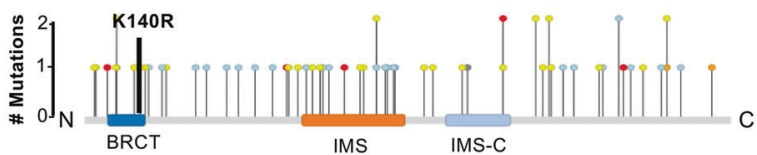

TDG1

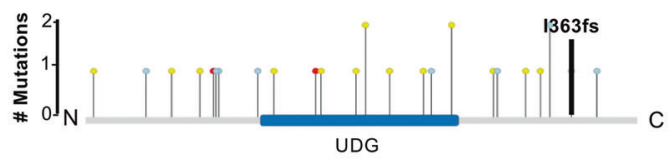

O Synonymous $O$ missense $O$ splice $O$ Truncating

former-smokers exhibited a wide range of mutation burdens $(3.3-43.7 / \mathrm{Mb})$ and smoking mutation signatures $(0-57 \%)$, despite over 10 pack-years of exposure in all but one case
(Fig. 3a). With the caveat that this observation is based on a small number of cases, this variation in mutation burden attributable to smoking was puzzling given the 
Fig. 3 Germline and somatic mutations in DNA repair genes and somatic mutation signatures. a Cases are listed according to smoking status, then by the fraction of the somatic mutation burden attributable to smoking. Pack-year (PY) exposure and mutations per $\mathrm{Mb}$ are indicated. Variants in DNA repair genes are depicted in oncoprint format. b Examples of germline mutations in DNA repair genes associated with tumor loss-of-heterozygosity. Needle plots generated by IntOGen depict somatic mutations in cancer. Protein domains are indicated using Pfam nomenclature. c Quantification of smoking signature and tumor mutation burden in tumors from patients with wild type (WT, $n=6$ ) or germline mutations (Mutant, $n=10$ ) in the DNA repair genes shown in Fig. 3a. Welch's $t$-test, two tailed. df degrees of freedom, NS not significant, LUAD lung adenocarcinoma, LUSC lung squamous cell carcinoma, SCLC small cell lung cancer

overwhelming evidence of a correlation between pack-year exposure and a smoking-related somatic signature in lung cancer [20]. Given the genotoxic effects of smoking on airway epithelial cells, and the importance of components of the DNA repair machinery in repairing this damage [21], we hypothesized that the degree of smoking-related DNA damage in each tumor may be related to germline variants in DNA repair genes.

We separately analyzed the germline WGS data from all 20 patients in the study without reference to the somatic mutations detected in the corresponding tumor samples. In 16 cases, we identified 30 potentially significant germline variants in DNA repair genes (Supplementary Table S3; Supplementary Data File S4). A subset of these genes is associated with repair of DNA damage induced by smoking, including ERCC5, ERCC8, LIG4, MUTYH, NEIL1, POLB, RECQL4, REV1, and TDG [21-23] (Fig. 3b; Supplementary Table S3). Although smoking can induce a range of genotoxic effects, these genes are of interest because they have been implicated in the repair of tobacco carcinogen adducts or oxidation-induced single-strand breaks. As shown in Fig. 3a, tumors with the strongest tobacco mutation signature occurred in patients with germline variants in these genes associated with somatic LOH. In addition, two hypermutated tumors with strong tobacco signatures harbored somatic mutations in LIG4 (LUAD5) and ERCC5 (LUSC3) (Fig. 3a). Considering all 17 ever-smokers as a group, the presence of a mutation in one or more of these genes was associated with a greater fraction of the somatic signature attributable to smoking $(P$ $<0.05$ ) but not overall mutation burden (Fig. 3c).

To validate this observation, we analyzed 576 non-SCLC cases in TCGA dataset without oncogenic mutations in EGFR or EML4-ALK rearrangements in which pack-year cigarette exposure was available (Supplementary Data File S5). Neither somatic mutation burden, smoking somatic signature percentage, or the number of variants potentially caused by smoking correlated with smoking history (Supplementary Figure S5). Analysis of heterozygous germline events in 48 DNA repair genes likely to be associated with smoking-induced DNA damage (Supplementary Data File
S2) identified 393 variants in 262 patients. Comparison of the estimated number of smoking-related variants per packyear with these germline variants identified a group of patients with minimal smoking histories and large somatic tobacco mutation burdens with germline events in genes identified in our EBUS-TBNA cohort (ERCC5, NEIL1, REV3L, REV1), as well as the NEIL1 ortholog NEIL3 (Fig. 4). In the patient with the highest tobacco mutation burden per pack-year, we detected variants in four genes, ERCC1, NEIL1, NEIL3, and REV3L (Fig. 4). By contrast, we did not detect germline variants in a group of patients with very low somatic smoking burdens, despite a heavy smoking history (Fig. 4a, b). Consistent with our data in the EBUS-TBNA cohort, patients with one or more germline variants in this gene list had tumors with increased numbers of smoking variants per pack-year, and a higher percentage of somatic smoking signature (Fig. 4c). Rather than directly contributing to the risk of lung cancer, these data suggest that the smoking mutation signature may be the result of an interaction between damaging germline variants in specific DNA repair genes and cigarette exposure.

\section{Phylogenetic models of lung cancer metastasis}

We prioritized resolving the degree of heterogeneity by focussing on comparisons between primary tumors and their matched metastases in 27 samples from 10 cases sequenced at an average depth of $150 \times($ Fig. 5a). Additional data from cases analyzed with single-region sequencing are shown in Supplementary Figure S6. By combining genomewide analysis of private and shared single nucleotide variants (SNVs) and insertions/deletions (Indels) with manually curated variants in known cancer driver genes (Supplementary Data File S2), we generated phylogenetic reconstructions for each patient (Fig. 5). In all cases, this analysis revealed that the last common ancestor accumulated a very large number of mutations, regardless of histotype or smoking history, when compared with private variants in the corresponding primary tumor and its derivative metastases (Fig. 5a). Although single-site sampling of all but two of the primary tumors in our study means that we cannot comprehensively model intratumor heterogeneity, these data suggest that metastatic lesions are not highly divergent from the primary tumor.

In a parallel analysis, we considered the degree of heterogeneity based on shared and private copy number events (Fig. 5b). Consistent with prior observations [10, 11], considerable heterogeneity in CNV was observed, suggesting that ongoing chromosomal instability is important in generating clonal diversity within primary tumors, and between primary tumors and their derivative metastases [2]. However, diversity based on CNV was not universal, with three cases (LUAD3, LUAD6, and SCLC2) exhibiting 
a

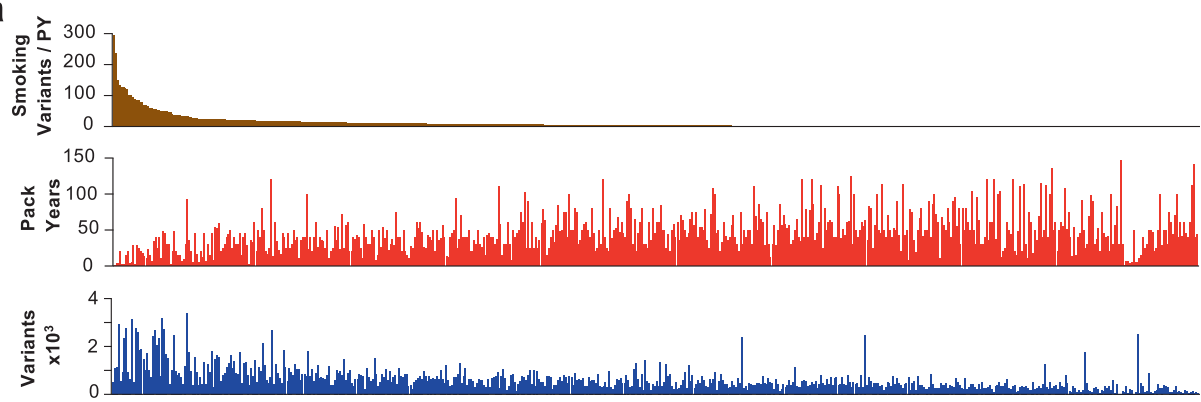

b

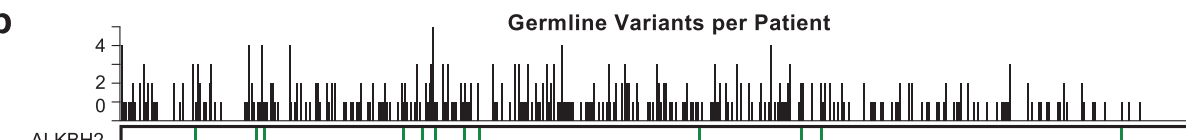

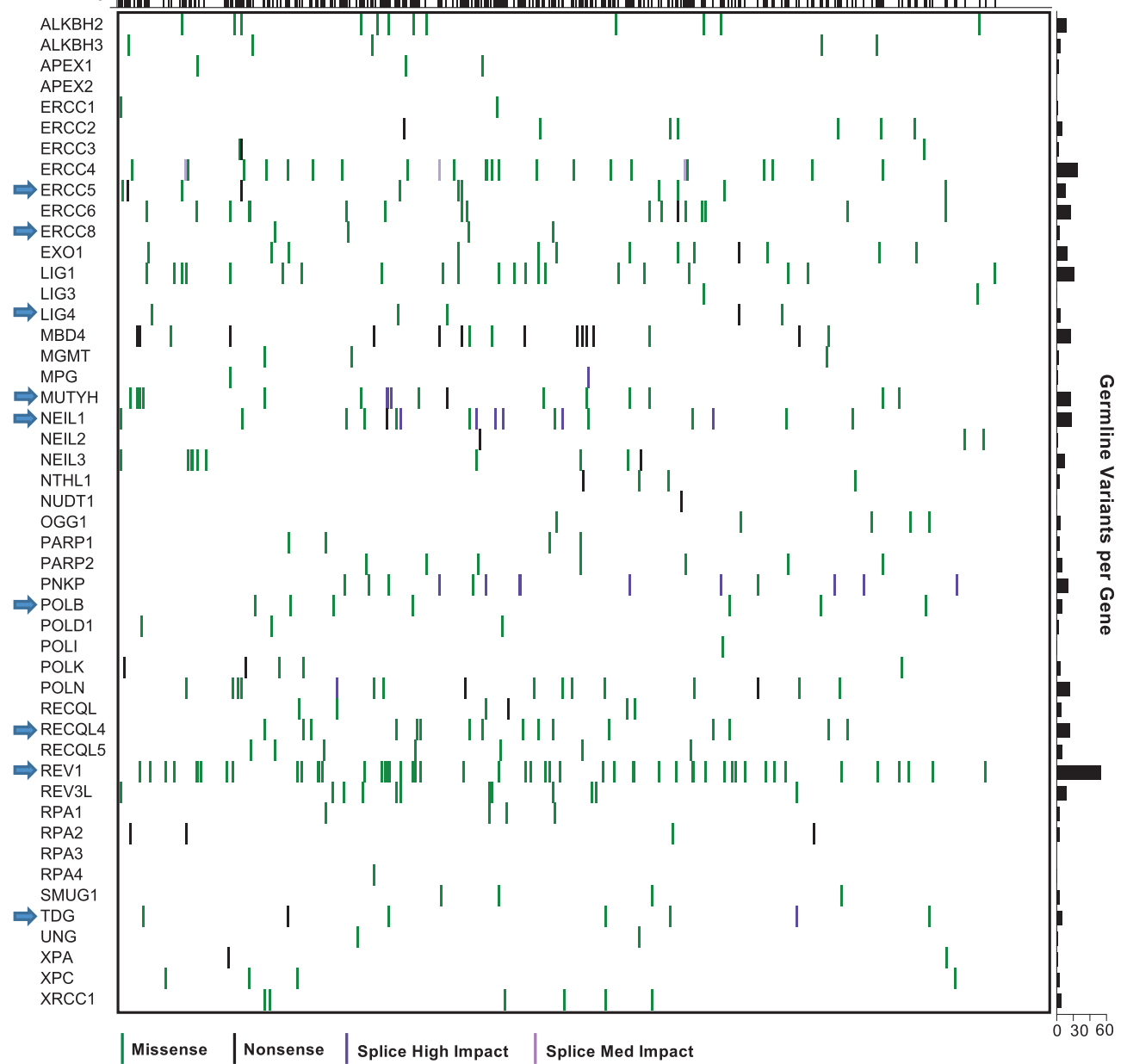

C

Smoking Variants / PY

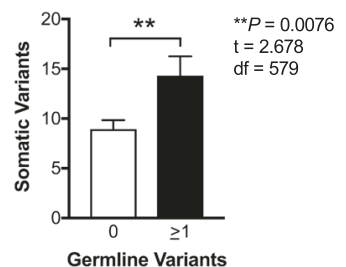

Smoking Signature \%

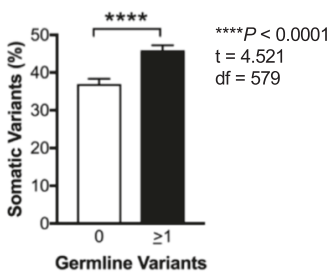

Smoking Variants

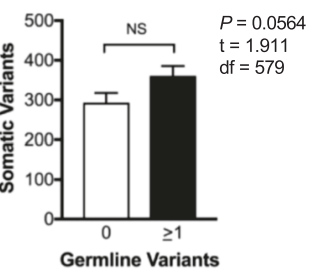

Total Variants
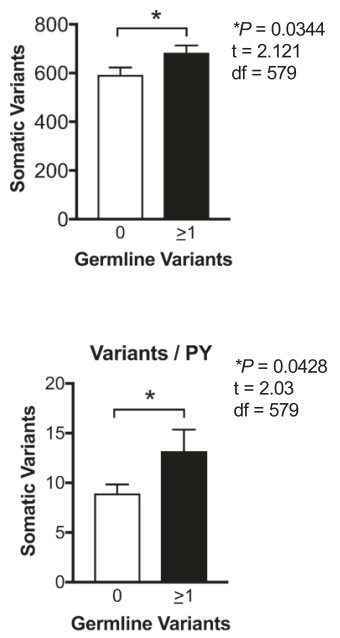

Fig. 4 Analysis of somatic mutation patterns and germline variants of TCGA non-small cell lung cancer data. a Somatic mutation burden in cases arranged by the number of smoking signature variants per pack-year (PY) exposure. Matched data for each case indicating total pack-years and total somatic variants are shown directly below. b Heterozygous germline variants in DNA repair genes potentially

remarkable similarity between primary and metastatic tumors (Fig. 5b). These data support the idea that genomic heterogeneity in lung cancer is largely driven by $\mathrm{CNV}$, but it may not be a universal mechanism for generating clonal diversity. linked to the repair of smoking-induced DNA damage arranged in the same order as above. Genes identified in the EBUS-TBNA cohort are labeled with an arrow. c Comparison of somatic mutation patterns between cases without germline variants $(n=314)$, or cases with one or more variants $(n=262)$. Mean \pm SEM, unpaired $t$-test, two tailed. $\mathrm{df}$ degrees of freedom, NS not significant

\section{Somatic signatures in private mutations}

In early-stage lung cancer, mutational diversity following the establishment of the last common ancestor has been associated with a somatic signature consistent with the 
a

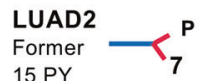

$\underset{7}{\text { LUAD1 }} \underset{20 \mathrm{PY}}{\mathrm{P}}$
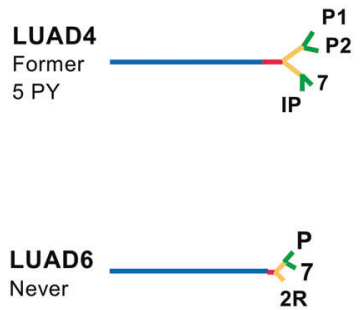

SCLC2

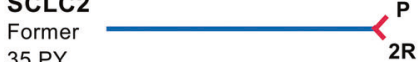

$35 \mathrm{PY}$

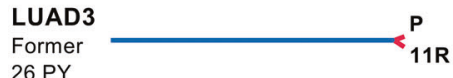

SCLC1

Current

$35 \mathrm{PY}$
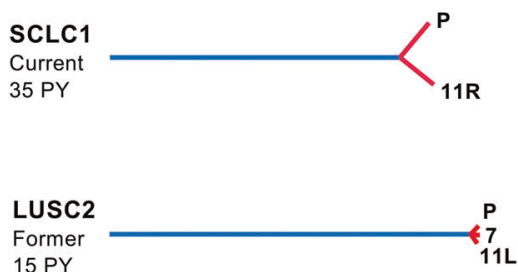

\section{LUAD5}

Current $<_{4 \mathrm{R}}^{\mathrm{P}}$

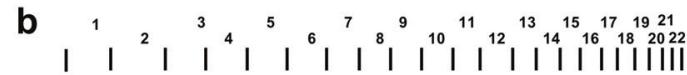

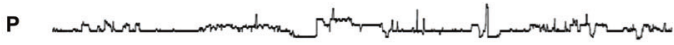

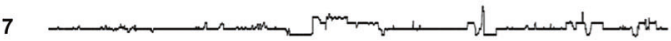

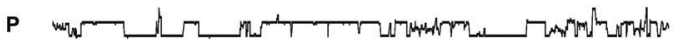

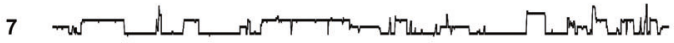

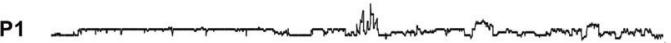

P2

(1)

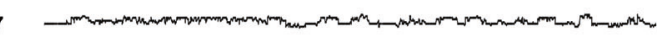

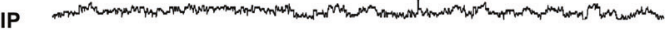

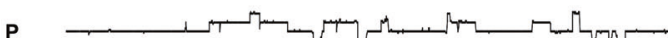

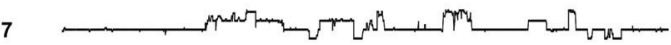

2R

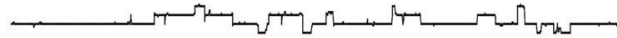

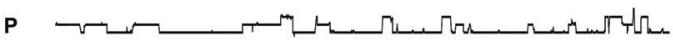

$2 \mathrm{R} \longrightarrow$ -

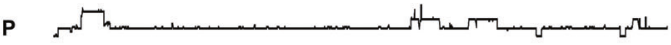

11L

P

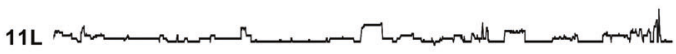

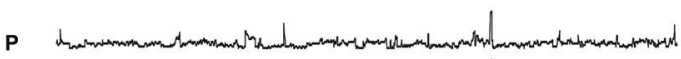

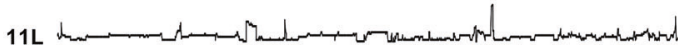

7

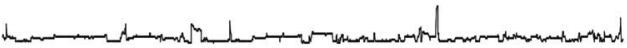

$P$ S

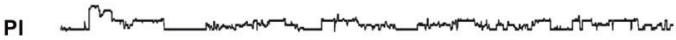

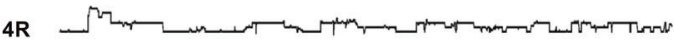
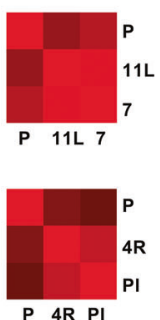

P 4R PI

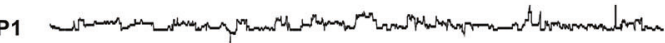

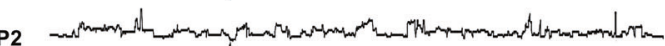

4L

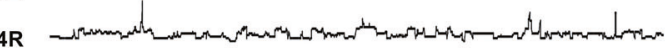

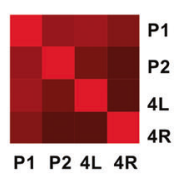

Former

$45 \mathrm{PY}$

\begin{tabular}{|c|c|c|c|c|c|c|c|}
\hline \multirow{3}{*}{ Variants } & \multirow{2}{*}{20,000} & \multicolumn{5}{|c|}{ Coefficient of Determination $\left(\mathrm{R}^{2}\right)$} & \\
\hline & & | & & & & & \\
\hline & & 0 & 0.2 & 0.4 & 0.6 & 0.8 & 1 \\
\hline
\end{tabular}

Fig. 5 Progression model of lung cancers reconstructed from the analysis of shared and private variants. The smoking status and packyear (PY) exposure for each case is listed. a SNVs and Indel counts shown to scale. b CNV plots scaled per patient for each sample. To the right, heat maps depict heterogeneity in copy number variation between samples in each case. $\mathrm{P}$ primary tumor; metastases are indicated by lymph node station except for Pl (pleura) and IP (intrapulmonary). LUAD lung adenocarcinoma, LUSC lung squamous cell carcinoma, SCLC small cell lung cancer 


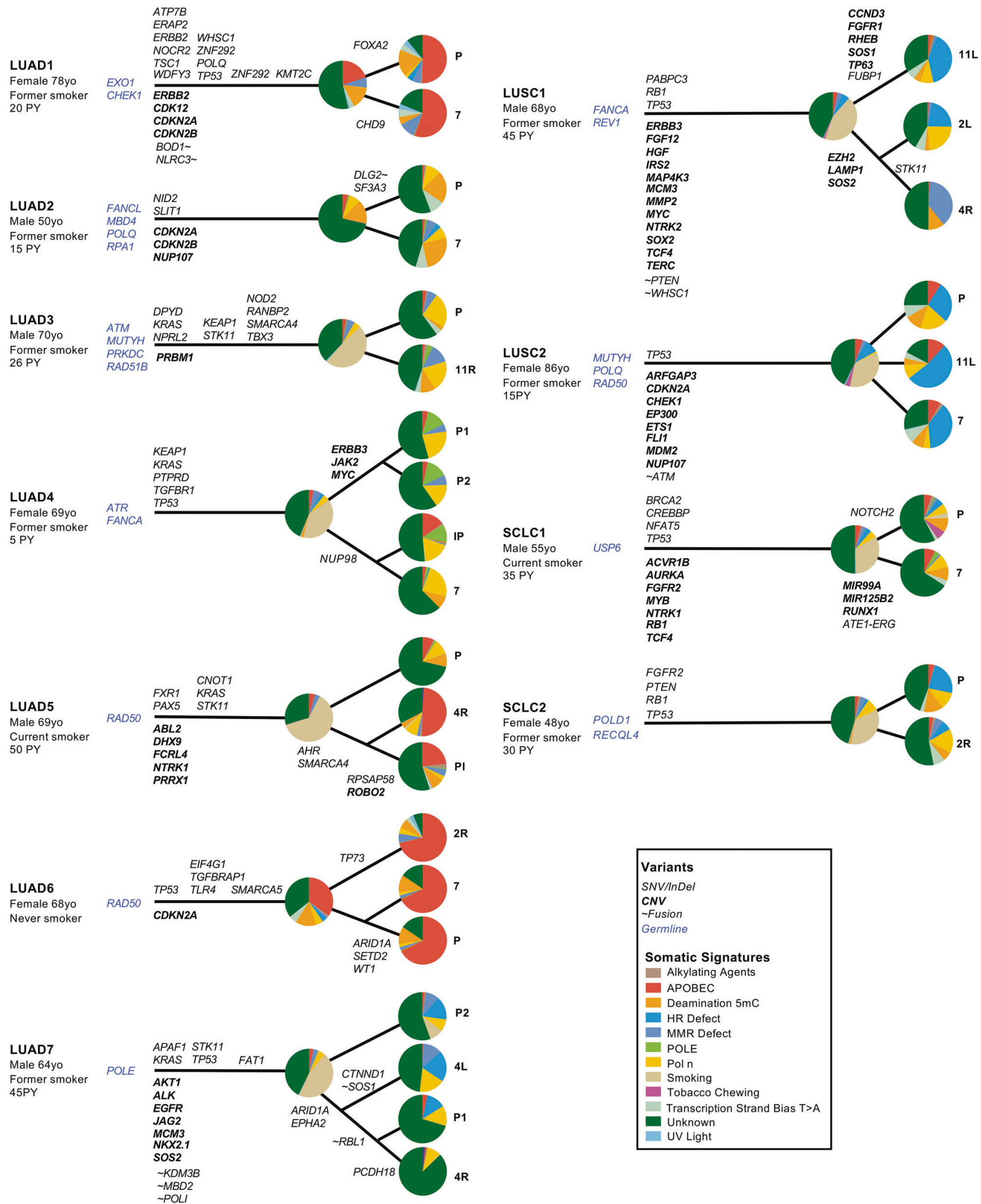

Fig. 6 Lung cancer progression models derived from multi-region whole-genome sequencing. Somatic variants by affected genes are shown from left-right according to variant allele frequency. Germline variants are shown immediately to the left of the putative point of origin of each tumor. Somatic signature pie charts depict events in the

common ancestor and private to each site. P primary tumor; metastases are indicated by lymph node station except for Pl (pleura) and IP (intrapulmonary). LUAD lung adenocarcinoma, LUSC lung squamous cell carcinoma, SCLC small cell lung cancer 

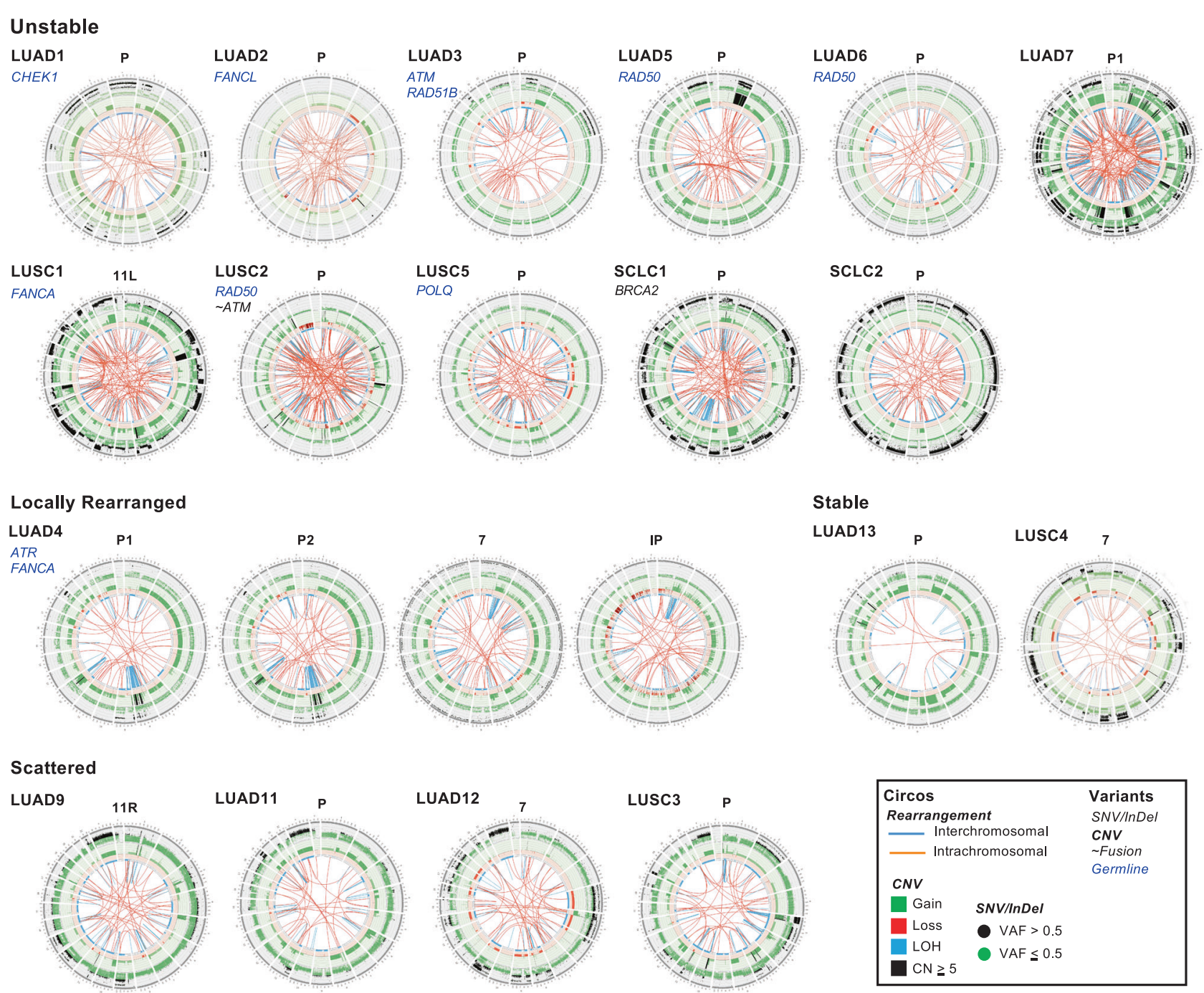

Fig. 7 Circos plots from samples analyzed by multi-region and singleregion WGS with patterns of structural variants consistent with unstable, locally rearranged, scatted and stable genomic signatures. P

primary tumor; metastases are indicated by lymph node station except for Pl (pleura) and IP (intrapulmonary). LUAD lung adenocarcinoma, LUSC lung squamous cell carcinoma, SCLC small cell lung cancer

activation of APOBEC enzymes [10, 11], an endogenous mutagenic process thought to be driven by inflammation [24]. To determine whether this process was seen in metastatic lung cancer, we analyzed somatic signatures in events private to both primary and metastatic tumors from the 11 patients with informative multi-region samples. Although we observed a strong APOBEC signature in private events in three LUAD cases (LUAD1, 2, 6; Fig. 6), private events in the remaining cases exhibited marked diversity in somatic signatures between patients, and between sampling sites within the same patient (Fig. 6). Excluding variants driven by as yet unknown mechanisms, private events in the remaining cases were dominated by patterns consistent with deamination of 5-mC (LUAD2; Fig. 6), Polymerase Eta (Poln) (LUAD3, 4; Fig. 6), or defects in homologous recombination (HR) (LUAD7, LUSC1, 2; Fig. 6). Unexpectedly, we also observed heterogeneity in private somatic signatures between samples within individual patients (Fig. 6), suggesting that the acquisition of SNV/Indels during progression from a common ancestor may also diverge along different paths.

To explain these results, we first scrutinized our mutation and $\mathrm{CNV}$ data to determine if private driver mutations or copy number events in genes relevant to each somatic signature could be identified. In each case, we could not identify a somatic mutation private to one or more sampling regions that might explain the wide spectrum of private somatic signatures we observed. This result raises the possibility that variants established in the common ancestor might influence the pattern of private mutations through interactions with intrinsic or extrinsic factors unique to each subclonal population. To test this idea, we considered truncal somatic events, as well as the germline variants in DNA repair genes identified in our germline analysis as 
potential mechanisms. In LUAD2, private mutations dominated by a signature for deamination of 5-mC (Fig. 6) were associated with germline variants in two genes functionally implicated in the repair of these mutations, $M D B 4$ [25] and RPA1 [26] (Fig. 6; Supplementary Table S3). Private events in two cases of LUSC were dominated by a signature suggestive of a defect in HR DNA repair (Fig. 6) and in both cases, germline variants with somatic $\mathrm{LOH}$ were detected in HR DNA repair genes FANCA in LUSC1 and $R A D 50$ in LUSC2. (Fig. 6; Supplementary Table S3; Supplementary Data File S4). Although we could not make such an association in cases with private event signatures dominated by APOBEC (Fig. 6; Supplementary Figure S5) or Poln (Figs. 6, 7), these data suggest that germline variants in a different subset of DNA repair genes may influence the acquisition of private somatic mutations during lung cancer progression, distinct from those associated with mutation burden in the founding clone.

\section{SVs and chromosomal instability}

The extent and complexity of somatic SVs in cancer can be associated with chromosomal instability, an important driver of tumor progression and genomic heterogeneity [27]. Moreover, SV patterns identified by WGS can distinguish tumors with transient periods of instability leading to focal chromosomal rearrangements from those with ongoing, genome-wide instability [13]. Most samples in our cohort demonstrated an SV pattern associated with highly unstable genomes that was shared between primary and metastatic tumors (Fig. 7; Supplementary Figure. S7). In all but three of these cases, we detected potentially deleterious germline variants in at least one DNA repair gene known to participate in the maintenance of chromosomal stability (CHEKI, FANCL, ATM, RAD51B, RAD50; Fig. 7; Supplementary Table S3). LUAD4 exhibited a distinct focal rearrangement pattern that diverged between primary and metastasis, also associated with germline variants in ATR and FANCA (Fig. 7). In the primary tumor, a prominent chromothriptic pattern was seen in chromosome 8 , whereas a distinct event was seen in chromosome 1 in both metastases (Fig. 7).

With the exception of LUSC2, which harbored a somatic LOF fusion in ATM (Figs. 2, 7; Supplementary Table S2), and SCLC1, which contained a somatic event in BRCA2, we did not detect shared or private events in genes associated with chromosomal instability. Additional Circos plots, along with an overview of additional genomic data for each case is shown in Supplementary Figure S8. In keeping with our somatic signature analysis, these data suggest that germline variants in DNA repair genes may also play a role in the progression of metastatic lung cancer through the generation of chromosomal instability.

\section{Discussion}

The propensity of lung cancer to spread within the chest prior to diagnosis is a major barrier to the delivery of curative therapy [28]. This long-standing clinical problem is likely to be driven by multiple factors, including the challenges associated with early screening, and the biological virulence of lung cancer. In addition, lung cancers have ready access to an abundant blood supply and complex lymphatic system that can promote metastasis at a very early-stage of tumor development [29]. Using EBUSTBNA sampling and high-depth WGS, we have demonstrated the genomic complexity and heterogeneity underlying the process of lung cancer metastasis in the chest. As part of this analysis, we detected mutations in tumorsuppressor genes not normally associated with lung cancer, novel LOF fusion events in critical tumor suppressors including PTEN and ATM, and passenger mutations in nondriver genes such as $A T P 7 B, E R A P 2$, and TLR2 that may have functional and/or therapeutic significance. The degree to which these findings differentiate early from late-stage lung cancer will require a comprehensive genomic evaluation in a large number of patients with metastatic disease.

The large number of variants identified through WGS allowed us to accurately map somatic signatures in our sample set. The degree of variation in the smoking signature of tumors in ever-smokers was surprising given the substantial pack-year exposure in most cases, and the high somatic mutation burden even in cases in which the somatic smoking signature was less dominant. Several potential explanations for this result can be considered. First, it is possible that inflammation caused by smoking, and its associated mutagenic effects, may predominate in some patients [30]. This idea is supported by the dominant APOBEC signature seen in three former-smokers. Second, it is possible that environmental effects other than smoking that drive lung cancer initiation in both former-smokers and never-smokers may be important, and that the interpretation of somatic signatures with respect to carcinogens such as second-hand smoke, radon, indoor air pollution, and occupational exposures [31] may need to be evaluated. Third, it is possible that germline variants contribute to the effect of smoking on the pattern of lung cancer mutations. Our data strongly suggest that germline variants in genes implicated in the repair of smoking-induced DNA damage may contribute to the somatic signatures observed in tumors from current- and former-smokers. If this assertion can be substantiated in larger prospective studies, it would suggest that such variants may only be penetrant in the setting of cigarette exposure, and may contribute to lung cancer risk only in smokers. 
Several studies have strongly implicated APOBEC activation as a driver of mutagenesis in lung cancer progression $[10,11]$, although this seems to be less prevalent in EGFR mutant LUAD in Asians [32]. With respect to mutations associated with metastatic lung cancer progression, our data suggest that this process is heterogeneous both within and between patients, with private somatic signatures of $\mathrm{HR}$ deficiency or deamination of $5-\mathrm{mC}$ present in addition to APOBEC signatures. In three cases, we identified germline variants that could be functionally associated with these signatures. Similarly, we observed a striking association between highly rearranged somatic genomes and germline variants in genes such as ATM, ATR, FANCA, and RAD51B. These results suggest that extrinsic factors unique to the environment of each metastasis may interact with germline variants to influence the mutation spectrum in metastatic disease. Large-scale prospective studies are needed to further validate these results in patients with distant metastatic disease. Given the connection between tumor mutation burden and the immune response [33], our data suggest that clinical trials in which the genomic heterogeneity in metastatic lung cancer in compared with the response to immunotherapy would be highly informative.

Given the poor prognosis of metastatic lung cancer, insights into the genomic evolution of this disease are urgently needed. In a recently published study, Um and colleagues used EBUS-TBNA sampling to perform multiregion exon capture and RNAseq analysis on six cases of advanced lung cancer [34]. In keeping with our results, mutations in critical genes, such as TP53 and $R B 1$, were shared across all sites in individual patients. They also observed variability in the degree of mutational heterogeneity, suggesting distinct models of clonal evolution in which metastatic spread occurs early or late during tumor progression. Although our results are consistent with previous observations that primary lung cancers are highly heterogeneous [10, 11], we find that mutational heterogeneity in metastatic disease is far more limited with respect to both driver and passenger genes, in keeping with a recent WGS study in four cases of metastatic pancreatic cancer [16]. Also in keeping with previous reports of multi-region sequencing in early-stage lung cancer, we found that metastatic heterogeneity is largely driven by CNV $[10,11]$.

Our data are consistent with two models of tumor evolution in metastatic lung cancer. In the first model, a slowly growing primary tumor progressively acquires a very large number of SNVs and Indels, followed much later by the establishment of metastases. However, such a model seems at odds with the clinical and biological behavior of lung cancer. Alternatively, our results could be explained by prolonged exposure of the airway to carcinogens, followed by rapid progression associated with chromosomal instability, accelerated growth, and metastasis. The airway epithelium is a low turnover cell population continuously exposed to environmental toxins, bacteria, viruses, and oxidative stress [35]. In addition, oncogenic mutations have been observed in the normal airway epithelium of eversmokers [36, 37], suggesting that mutant airway epithelial clones can persist for decades. Given the lack of ready access to well-defined precursor lesions in lung cancer research [38], detailed genomic analysis of normal airway epithelium in ever-smokers with lung cancer may provide critical insights into the early events driving the establishment and progression of lung cancer. More detailed prospective studies will be needed to address whether the histologic subtypes of lung cancer, particularly adenocarcinoma, relate to environmental exposure, smoking, and germline variants. Finally, our results suggest that gene-byenvironment interactions in the DNA repair pathway may influence the mutational pathogenesis of lung cancer during tumor initiation, progression and metastasis.

\section{Materials and methods}

\section{Subject cohort and sample processing}

Patients undergoing EBUS-TBNA for diagnosis and/or staging gave prospective written informed consent to participate in this study. The study was approved by the $\mathrm{St}$ Vincent's Hospital human research ethics committee protocol number SVH14-256. Once the on-site cytologist confirmed the diagnostic material had been obtained for clinical purposes, an extra sample was taken for research purposes, suspended in sterile saline, and placed on ice.

In the laboratory, each sample was disaggregated, centrifuged, and re-suspended in $450 \mu \mathrm{l}$ of PBS. Next, a $50 \mu \mathrm{l}$ aliquot was removed to generate a cytology smear to determine tumor cellularity of the research specimen. The cytology specimen was stained with DiffQuik, and then reviewed by a cytopathologist to estimate the fraction of the sample made up of tumor cells, as well as sample quality. Samples containing $>20 \%$ tumor cells were then processed for DNA extraction from the frozen cell suspension using the Qiagen (Hilden, Germany) DNEasy kit as per the manufacturer's instructions, and then checked for quality, purity, and integrity in preparation for WGS. Germline DNA was obtained using the same methodology from peripheral blood mononuclear cells.

\section{WGS}

Sequencing was carried out on the Illumina (San Diego, CA, USA) HiSeq X Ten platform with a paired-end read length of 150 bases. We sequenced all germline (peripheral blood buffy coat) samples to a minimum mean coverage of 
$30 \times$ and all tumor samples to a minimum mean coverage of $60 \times$. In cases where multi-region samples were available, samples with an inferred cellularity of $>15 \%$ were sequenced to an additional minimum mean coverage of $90 \times$, giving a total of at least $150 \times$.

\section{Bioinformatics}

The analysis pipeline was conducted using the Seave platform [39]. Somatic SNVs and Indels were called using Strelka v2.0.17.strelka1 [40] and the GATK Best Practices workflow [41]. Final variants were annotated for their impact on the genome using Variant Effect Predictor v87 [42]. Variants were filtered using a CADD [42] scaled score of $>2$ (if available) and a maximum allele frequency of $1 \%$ for somatic variants and $2 \%$ for germline variants in each of ExAC [43]. Somatic variant filtration was performed using two parallel approaches. First, we generated a consensus cancer gene driver list that includes pan-cancer driver genes [44], and putative lung cancer driver genes [7-9] (Supplementary Data File S1). Candidates were then identified by filtering on Strelka QSS/QSI >15, impact severity, and PolyPhen/PROVEAN/SIFT prediction, and then annotated using PubMed, IntOGen [45] and Varsome (www.varsome. com). In the second approach, we queried all genes and identified additional LOF candidates by filtering using the same filtration approach with the added criteria of evidence for somatic LOH. Candidates were then manually annotated by searching for evidence of a potential link between cancer pathogenesis or therapeutic responses using PubMed.

Germline variants were analyzed using a consensus list of DNA repair genes generated by searching the Gene Ontology database [46] (Supplementary Data File S2). Missense variants were further filtered according to the following criteria: (ClinVar annotation pathogenic or likely pathogenic) OR (variant in a Pfam domain AND defined as damaging by PROVEAN OR SIFT or PolyPhen) OR (defined as damaging in at least two of either PROVEAN, SIFT or PolyPhen). LOH events were confirmed by comparing the variant allele frequency (VAF) in germline and tumor samples with inferred tumor cellularity. Somatic signatures were analyzed using the SomaticSignatures $\mathrm{R}$ package (v2.6.0) [47]. Somatic signatures for TCGA LUAD and LUSC data were determined using somatic variants called by MuTect 2 and obtained from TCGA. Variant call files (VCFs) were subset to autosomal chromosomes and PASS variants were used only, all other analysis was identical to the EBUS-TBNA data.

Somatic CNVs were identified using Sequenza v2.1.0 [48] using a bin size of 200 and a minimum total depth of 20. Candidate CNV events were identified by filtering for estimated copy number 0 or $>6$ and segment size $>20 \mathrm{~kb}$. Events were then verified by identifying a corresponding event using Manta v0.27.1 [49], and manually with IGV. Candidates were then annotated by searching the PubMed, COSMIC [50], and CBioPortal [51]. SV calling and fusion analysis was performed with Manta v0.27.1 [49] and Oncofuse v1.0.9b2 [52]. Further details of describing the bioinformatic analysis are presented in Supplementary Information. Sequencing data are available at the European Nucleotide Archive (https://www.ebi.ac.uk/ena) under accession number PRJEB28616.

Acknowledgements This work was supported by the Victorian Cancer Agency, (TS10-01), the Petre Foundation, the Victorian Government's Operational Infrastructure Support Program, the Baxter Charitable Foundation, the St Vincent's Clinic Foundation, Tour de Cure, the Australian Government NHMRC IRIISS, the Victorian State Government Operational Infrastructure Support, the Australian Cancer Therapeutics CRC (TLL), the Australian Cancer Research Foundation, an NHMRC Career Development Fellowship (GNT1063914, DJG), a Victoria Cancer Agency Mid-Career Fellowship (MCRF17014, JEC), a Viertel Foundation Senior Medical Researcher Fellowship (M-LAL), a Cancer Institute NSW Early Career Fellowship (13ECF/1-46) and NSW Health Early-Mid-Career Fellowship (MJC), the Kinghorn Foundation (MJC, VG), and an NHMRC Senior Research Fellowship (GNT546107, DNW). We thank the Victorian Cancer Biobank for technical support.

Author contributions The authors made the following contributions: conception and design: TLL, M-LA-L, MJC, DNW; development of methodology: TLL, VG, DPP, M.R.D, M-LA-L, MJC, DNW; acquisition and interpretation of data: TLL, VG, KDM, ES, VC, RAM, AF, PAR, BK, DJG, AS, FJR, JEC, ATP, M-LA-L, MJC, DNW; patient recruitment, consent, and sample acquisition: TLL, DPP, ES, VC, AH, LBB, BRJ, MA, VB, VG, DNW; administrative, technical or material support: AG-R, KDM, VC, RAM, WSNJ, AS, JEC, DNW; writing, review and/or revision of the manuscript: all authors; study supervision: MLL, MJC, DNW.

\section{Compliance with ethical standards}

Conflict of interest The authors declare that they have no conflict of interest.

Open Access This article is licensed under a Creative Commons Attribution 4.0 International License, which permits use, sharing, adaptation, distribution and reproduction in any medium or format, as long as you give appropriate credit to the original author(s) and the source, provide a link to the Creative Commons license, and indicate if changes were made. The images or other third party material in this article are included in the article's Creative Commons license, unless indicated otherwise in a credit line to the material. If material is not included in the article's Creative Commons license and your intended use is not permitted by statutory regulation or exceeds the permitted use, you will need to obtain permission directly from the copyright holder. To view a copy of this license, visit http://creativecommons. org/licenses/by/4.0/.

\section{References}

1. McGranahan N, Swanton C. Biological and therapeutic impact of intratumor heterogeneity in cancer evolution. Cancer Cell. 2015;27:15-26. 
2. Maley CC, Aktipis A, Graham TA, Sottoriva A, Boddy AM, Janiszewska M, et al. Classifying the evolutionary and ecological features of neoplasms. Nat Rev Clin Oncol. 2017;17:605-19.

3. Amirouchene-Angelozzi N, Swanton C, Bardelli A. Tumor evolution as a therapeutic target. Cancer Discov. 2017;7:805-17.

4. Turajlic S, Swanton C. Metastasis as an evolutionary process. Science. 2016;352:169-75.

5. Makohon-Moore AP, Gerold JM, Bozic I, Chatterjee K, Iacobuzio-Donahue CA, Vogelstein B, et al. Reconstructing metastatic seeding patterns of human cancers. Nat Commun. 2017;8:1-10.

6. Chansky K, Detterbeck FC, Nicholson AG, Rusch VW, Vallières $\mathrm{E}$, Groome P, et al. The IASLC lung cancer staging project: external validation of the revision of the TNM stage groupings in the ighth edition of the TNM classification of lung cancer. J Thorac Oncol. 2017;12:1109-21.

7. Cancer Genome Atlas Research Network, Campbell JD, Brooks AN, Berger AH, Lee W, Chmielecki J, et al. Comprehensive molecular profiling of lung adenocarcinoma. Nature. 2014;511:543-50.

8. Cancer Genome Atlas Research Network, Lawrence MS, Voet D, Jing R, Cibulskis K, McKenna A, et al. Comprehensive genomic characterization of squamous cell lung cancers. Nature. 2012;489:519-25.

9. George J, Lim JS, Jang SJ, Cun Y, Ozretić L, Kong G, et al. Comprehensive genomic profiles of small cell lung cancer. Nature. 2015;524:47-53.

10. Jamal-Hanjani $\mathrm{M}$, Wilson GA, McGranahan $\mathrm{N}$, Birkbak NJ, Watkins TBK, Veeriah S, et al. Tracking the evolution of nonsmall-cell lung cancer. N Engl J Med. 2017;376:2109-21.

11. Zhang J, Fujimoto J, Zhang J, Wedge DC, Song X, Zhang J, et al. Intratumor heterogeneity in localized lung adenocarcinomas delineated by multiregion sequencing. Science. 2014;346:256-9.

12. Leong TL, Marini KD, Rossello FJ, Jayasekara SN, Russell PA, Prodanovic Z, et al. Genomic characterisation of small cell lung cancer patient-derived xenografts generated from endobronchial ultrasound-guided transbronchial needle aspiration specimens. PLoS ONE. 2014;9:e106862.

13. Waddell N, Pajic M, Patch A-M, Chang DK, Kassahn KS, Bailey $\mathrm{P}$, et al. Whole genomes redefine the mutational landscape of pancreatic cancer. Nature. 2015;518:495-501.

14. Meienberg J, Bruggmann R, Oexle K, Matyas G. Clinical sequencing: is WGS the better WES? Hum Genet. 2016;135:35962.

15. Alioto TS, Buchhalter I, Derdak S, Hutter B, Eldridge MD, Hovig $\mathrm{E}$, et al. A comprehensive assessment of somatic mutation detection in cancer using whole-genome sequencing. Nat Commun. 2015;6:10001.

16. Makohon-Moore AP, Zhang M, Reiter JG, Bozic I, Allen B, Kundu $D$, et al. Limited heterogeneity of known driver gene mutations among the metastases of individual patients with pancreatic cancer. Nat Rev Clin Oncol. 2017;49:358-66.

17. Cardarella S, Johnson BE. The impact of genomic changes on treatment of lung cancer. Am J Respir Crit Care Med. 2013;188:770-5.

18. Adamo P, Ladomery MR. The oncogene ERG: a key factor in prostate cancer. Oncogene. 2016;35:403-14.

19. Chen X, Bahrami A, Pappo A, Easton J, Dalton J, Hedlund E, et al. Recurrent somatic structural variations contribute to tumorigenesis in pediatric osteosarcoma. Cell Rep. 2014;7:10412

20. Alexandrov LB, Ju YS, Haase K, Van Loo P, Martincorena I, NikZainal S, et al. Mutational signatures associated with tobacco smoking in human cancer. Science. 2016;354:618-22.

21. Wu X, Zhao H, Suk R, Christiani DC. Genetic susceptibility to tobacco-related cancer. Oncogene. 2004;23:6500-23.
22. Shields PG. Molecular epidemiology of smoking and lung cancer. Oncogene. 2002;21:6870-6.

23. Wiencke JK. DNA adduct burden and tobacco carcinogenesis. Oncogene. 2002;21:7376-91.

24. Campbell BB, Light N, Fabrizio D, Zatzman M, Fuligni F, de Borja R, et al. Comprehensive analysis of hypermutation in human. Cancer Cell. 2017;171:1042-.e10.

25. Yu AM, Calvo JA, Muthupalani S, Samson LD. The Mbd4 DNA glycosylase protects mice from inflammation-driven colon cancer and tissue injury. Oncotarget. 2016;7:28624-36.

26. Chaudhuri J, Khuong C, Alt FW. Replication protein A interacts with AID to promote deamination of somatic hypermutation targets. Nature. 2004;430:992-8.

27. Bakhoum SF, Landau D-A. Chromosomal instability as a driver of tumor heterogeneity and evolution. Cold Spring Harb Perspect Med. 2017;7:a029611.

28. Detterbeck FC, Boffa DJ, Tanoue LT. The new lung cancer staging system. Chest. 2009;136:260-71.

29. Jin X-R, Zhu L-Y, Qian K, Feng Y-G, Zhou J-H, Wang R-W, et al. Circulating tumor cells in early stage lung adenocarcinoma: a case series report and literature review. Oncotarget. 2017;8:23130-41.

30. Houghton AM. Mechanistic links between COPD and lung cancer. Nat Rev Cancer. 2013;13:233-45.

31. Samet JM, Avila-Tang E, Boffetta P, Hannan LM, Olivo-Marston $\mathrm{S}$, Thun MJ, et al. Lung cancer in never smokers: clinical epidemiology and environmental risk factors. Clin Cancer Res. 2009;15:5626-45.

32. Nahar R, Zhai W, Zhang T, Takano A, Khng AJ, Lee YY, et al. Elucidating the genomic architecture of Asian EGFR-mutant lung adenocarcinoma through multi-region exome sequencing. Nat Commun. 2018;9:1-11.

33. Zappasodi R, Merghoub T, Wolchok JD. Emerging concepts for immune checkpoint blockade-based combination therapies. Cancer Cell. 2018;33:581-98.

34. Um SW, Joung JG, Lee H, Kim H, Kim KT, Park J, et al. Molecular evolution patterns in metastatic lymph nodes reflect the differential treatment response of advanced primary lung cancer. Cancer Res. 2016;76:6568-76.

35. Grainge CL, Davies DE. Epithelial injury and repair in airways diseases. Chest. 2013;144:1906-12.

36. Kadara H, Wistuba II. Field cancerization in non-small cell lung cancer: implications in disease pathogenesis. Proc Am Thorac Soc. 2012;9:38-42.

37. Jakubek Y, Lang W, Vattathil S, Garcia M, Xu L, Huang L, et al. Genomic landscape established by allelic imbalance in the cancerization field of a normal appearing airway. Cancer Res. 2016;76:3676-83.

38. Kadara H, Scheet P, Wistuba II, Spira AE. Early events in the molecular pathogenesis of lung cancer. Cancer Prev Res (Phila). 2016;9:518-27.

39. Gayevskiy V, Roscioli T, Dinger ME, Cowley MJ. Seave: a comprehensive web platform for storing and interrogating human genomic variation. Bioinformatics. 2018. https://doi.org/10.1093/ bioinformatics/bty540

40. Saunders CT, Wong WSW, Swamy S, Becq J, Murray LJ, Cheetham RK. Strelka: accurate somatic small-variant calling from sequenced tumor-normal sample pairs. Bioinformatics. 2012;28:1811-7.

41. McKenna A, Hanna M, Banks E, Sivachenko A, Cibulskis K, Kernytsky A, et al. The genome analysis toolkit: a MapReduce framework for analyzing next-generation DNA sequencing data. Genome Res. 2010;20:1297-303.

42. McLaren W, Gil L, Hunt SE, Riat HS, Ritchie GRS, Thormann A, et al. The Ensembl variant effect predictor. Genome Biol. 2016;17:122. 
43. Kircher M, Witten DM, Jain P, O'Roak BJ, Cooper GM, Shendure J. A general framework for estimating the relative pathogenicity of human genetic variants. Nat Rev Clin Oncol. 2014;46:310-5.

44. Lek M, Karczewski KJ, Minikel EV, Samocha KE, Banks E, Fennell T, et al. Analysis of protein-coding genetic variation in 60,706 humans. Nature. 2016;536:285-91.

45. Gonzalez-Perez A, Perez-Llamas C, Deu-Pons J, Tamborero D, Schroeder MP, Jene-Sanz A, et al. IntOGen-mutations identifies cancer drivers across tumor types. Nat Method. 2013;10:1081-2.

46. The Gene Ontology Consortium. Expansion of the gene ontology knowledgebase and resources. Nucl Acid Res. 2017;45:D331-8.

47. Gehring JS, Fischer B, Lawrence M, Huber W. SomaticSignatures: inferring mutational signatures from singlenucleotide variants. Bioinformatics. 2015;31:3673-5.
48. Favero F, Joshi T, Marquard AM, Birkbak NJ, Krzystanek M, Li Q, et al. Sequenza: allele-specific copy number and mutation profiles from tumor sequencing data. Ann Oncol. 2015;26:64-70.

49. Chen X, Schulz-Trieglaff O, Shaw R, Barnes B, Schlesinger F, Källberg $\mathrm{M}$, et al. Manta: rapid detection of structural variants and indels for germline and cancer sequencing applications. Bioinformatics. 2016;32:1220-2.

50. Forbes SA, Beare D, Boutselakis H, Bamford S, Bindal N, Tate J, et al. COSMIC: somatic cancer genetics at high-resolution. Nucl Acid Res. 2017;45:D777-83.

51. Gao J, Aksoy BA, Dogrusoz U, Dresdner G, Gross B, Sumer SO, et al. Integrative analysis of complex cancer genomics and clinical profiles using the cBioPortal. Sci Signal. 2013;6:pl1.

52. Shugay M, Ortiz de Mendíbil I, Vizmanos JL, Novo FJ. Oncofuse: a computational framework for the prediction of the oncogenic potential of gene fusions. Bioinformatics. 2013;29:2539-46.

\section{Affiliations}

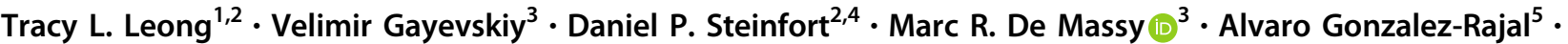
Kieren D. Marini ${ }^{6}$. Emily Stone ${ }^{6,7}$. Venessa Chin ${ }^{5,8,9} \cdot$ Adrian Havryk $^{7,8}$ - Marshall Plit ${ }^{7,8}$ - Louis B. Irving ${ }^{2,4}$. Barton R. Jennings ${ }^{10} \cdot$ Rachael A. McCloy ${ }^{5}$ W. Samantha N. Jayasekara ${ }^{6} \cdot$ Muhammad Alamgeer $^{6} \cdot$ Vishal Boolell $^{6}$. Andrew Field ${ }^{8,11}$. Prudence A. Russell ${ }^{12}$. Beena Kumar ${ }^{13}$. Daniel J. Gough ${ }^{6,14}$ - Anette Szczepny ${ }^{6}$. Vinod Ganju $\mathbb{1}^{6,14} \cdot$ Fernando J. Rossello ${ }^{15,16}$ • Jason E. Cain ${ }^{6,14}$ - Anthony T. Papenfuss ${ }^{17,18,19}$ • Marie-Liesse Asselin-Labat $\mathbb{D}^{1,2} \cdot$ Mark J. Cowley ${ }^{3,8,20} \cdot$ D. Neil Watkins ${ }^{5,7,8,9}$

1 ACRF Stem Cells and Cancer Division, The Walter and Eliza Hall Institute of Medical Research, Parkville, VIC 3050, Australia

2 Department of Medical Biology, University of Melbourne, Parkville, VIC 3050, Australia

3 The Kinghorn Centre for Clinical Genomics, Garvan Institute of Medical Research, Darlinghurst, NSW 2010, Australia

4 Department of Respiratory Medicine, Royal Melbourne Hospital, Parkville, VIC 3050, Australia

5 The Kinghorn Cancer Centre, Garvan Institute of Medical Research, Darlinghurst, NSW 2010, Australia

6 The Hudson Institute of Medical Research, Clayton, VIC 3168 , Australia

7 Department of Thoracic Medicine, St Vincent's Hospital, Darlinghurst, NSW 2010, Australia

8 St Vincent's Clinical School, UNSW Sydney, Darlinghurst, NSW 2010, Australia

9 Department of Medical Oncology, St Vincent's Hospital, Darlinghurst, NSW 2010, Australia

10 Department of Respiratory and Sleep Medicine, Monash Health, Clayton, VIC 3168, Australia

11 Department of Pathology, St Vincent's Hospital, Sydney, NSW

\section{0, Australia}

12 Department of Pathology, St Vincent's Hospital Melbourne, Fitzroy, VIC 3000, Australia

13 Department of Pathology, Monash Health, Clayton, VIC 3168, Australia

14 Department of Molecular and Translational Science, Faculty of Medicine, Nursing and Health Sciences, Monash University, Clayton, VIC, Australia

15 Department of Anatomy and Developmental Biology, Faculty of Medicine, Nursing and Health Sciences, Monash University, Clayton, VIC 3168, Australia

16 Australian Regenerative Medicine Institute, Monash University, Clayton, VIC 3168, Australia

17 Computational Cancer Biology Program, Peter MacCallum Cancer Centre, Melbourne, VIC 3000, Australia

18 Sir Peter MacCallum Department of Oncology, The University of Melbourne, Parkville, VIC 3052, Australia

19 Bioinformatics Division, The Walter \& Eliza Hall Institute of Medical Research, Parkville, VIC 3052, Australia

20 Children's Cancer Institute, Kensington, NSW 2750, Australia 